\title{
Sexualität, Erotik, Intimität
}

\author{
Sabine Kern · Christian Stadler
}

Nach den Heften zum Thema Paare und zum Thema Begegnung, Tele, Beziehung kommt nun ein drittes Heft, welches sich im psychodramatischen Kontext mit einem spezifischen Feld des persönlichen Lebens auseinandersetzt, mit Sexualität, Intimität und Erotik. Diese Themen auf die Bühne zu bringen ist für ProtagonistInnen und manchmal auch für TherapeutInnen nicht immer einfach, jedoch von großer Bedeutung, stellen sie doch zentrale menschliche Ausdrucksformen dar.

Neben AutorInnen, die den ZPS-LeserInnen bereits aus früheren Beiträgen bekannt sind, haben wir neue AutorInnen dafür gewinnen können, über dieses Thema zu schreiben. Alle miteinander zeichnen sich durch große Fachkompetenz in ihren Arbeitsgebieten aus. Zwei der Beiträge stammen von südeuropäischen AutorInnen (Portugal und Türkei), die damit auch den Aspekt der Kulturgebundenheit von sexuellen Ausdrucksformen und Störungen anklingen lassen.

Andreas Schulz befasst sich in seinem Beitrag mit den Voraussetzungen, die für das Gelingen einer erfüllenden und liebevollen Beziehung gegeben sein müssen. Als Grundstock dafür sieht der Autor das Vertrauen in die eigene Person und in den oder die PartnerIn. In seinen psychodramatischen Beratungen und Seminaren lässt Schulz die Paare den auf ihre Bedürfnisse und Wünsche zugeschnittenen Weg zwischen den Polen von Nähe und Distanz, der passenden Intensität an Intimität und den für sie stimmigen Grad an Treue erforschen. Durch psychodramatische Aufstellungen, Arbeiten mit Skulpturen und Vignetten wird den Paaren, aber auch Einzelpersonen, die an „Liebesverletzungen“ leiden, feinfühlig ein Raum eröffnet, in dem alte Rollenmuster durchforstet und mit neuen experimentiert werden kann.

Jutta Fürst und Hannes Krall plädieren in ihrem Artikel dafür, dem Thema Sexualität in der Therapieausbildung mehr Bedeutung zukommen zu lassen. Sie gehen davon aus, dass durch das Erwerben von Kompetenzen, wie zum Beispiel auf der Begegnungsbühne mit sexuellem Begehren umgegangen werden kann, mehr Sicherheit bei der Bearbeitung sexueller Themen entstehen und die Anzahl an sexuellen Übergriffen zwischen Thera-

\author{
Online publiziert: 08.02 .2012 \\ (C) VS Verlag für Sozialwissenschaften 2012 \\ C. Stadler $(\bowtie) \cdot$ S. Kern \\ Dachau, Deutschland \\ E-Mail: stadlercc@arcor.de \\ S. Kern \\ Wien, Österreich \\ E-Mail: sabine.kern@psychodramazentrum.at
}


peutInnen und KlientInnen verringert werden könnte. Nach einem kurzen Exkurs in die soziokulturelle Geschichte des Umgangs mit Sexualität beschreiben Fürst und Krall wodurch eine gesunde psychosexuelle Entwicklung gefördert werden könnte. Sie stellen zahlreiche, der Materie Leichtigkeit schenkende Übungen vor, die dem Thema Sexualität den ihr gebührenden Platz in der Therapieausbild verschaffen könnten.

Die beiden portugiesischen PsychodramatikerInnen Gabriela Moita und José Teixeira de Sousa gewähren uns Einblick in ein psychodramatisches Wochenendseminar für drei Paare, die in sexuellen Interaktionen Probleme bei der Penetration hatten. Da die herkömmliche sexualtherapeutische Behandlung den Vaginismus der betroffenen Frauen nicht zufriedenstellend lösen konnte, versuchten die beiden AutorInnen, sich die positiven Wirkmechanismen des Psychodramas zu Nutze zu machen. Durch den Einsatz von auf die Problematik einfallsreich zugeschnittenen psychodramatischen Arrangements und Techniken sowie der Nutzung des von den Beteiligten als entlastend erlebten Gruppensettings, konnte der Angstpegel der Betroffenen gesenkt und der soziale Druck vermindert werden, wodurch die Möglichkeit der sexuellen Vereinigung erleichtert wurde.

Arşaluys Kayir und Banu Aslantaş Ertekin streichen in ihrem Artikel die Bedeutung psychodramatischer Arrangements hervor, die in der Sexualtherapie der psychiatrischen Abteilung der Medizinischen Fakultät der Universität Istanbul eingesetzt werden. Im Anfangsteil stellen sie verschiedene Sexualstörungen und deren klassische therapeutische Behandlung vor. Sie legen in Folge einen Schwerpunkt auf die Problematik des Vaginismus, von dem in der sehr traditionell geprägten türkischen Gesellschaft viele Frauen betroffen sind. Das hier präsentierte umfangreiche Repertoire an speziellen psychodramatischen Interventionen zur Behandlung von Vaginismus, zeugt von der jahrzehntelangen Erfahrung der Autorinnen und wird sehr anschaulich von zahlreichern Fallbeispielen unterlegt.

Sonja Hintermeier geht in ihrem Artikel von der Annahme aus, dass die strukturellen Defizite von an Persönlichkeitsstörungen leidenden Personen, sich in ihrem Sexualverhalten verdichtet widerspiegeln. Nach einer Zusammenfassung psychodramatischer Theorie der Persönlichkeitsstörungen arbeitet sie anhand von plastischen Beispielen die charakteristischen Themen und Beziehungsgestaltungen der verschiedenen Persönlichkeitsstörungen heraus und setzt sie in Zusammenhang zu deren typischen Formen von sexuellen Interaktionen. Sie nutzt in ihrer therapeutischen Arbeit sexuelle Szenen, um die, der Persönlichkeitsstörung zugrundeliegenden, perfekten Ziele herauszuarbeiten und langsam zu integrieren. Dabei beschreibt sie ihr Vorgehen auf der inneren-, der Spiel-, aber auch auf der Begegnungsbühne auf sehr eindrucksvolle Weise.

Gabriele Denk berichtet in ihrem Beitrag wie Menschen mit geistiger und/oder körperlicher Behinderung bei der Entwicklung ihrer Rolle als sexuell aktive Menschen unterstützt werden können. Bezugnehmend auf die psychodramatische Rollen- und Entwicklungstheorie erklärt sie, auf welche Probleme und Hindernisse Menschen mit Behinderung bei der Entwicklung ihrer sexuellen Identität stoßen können. Sie gibt einen berührenden Einblick in ihre psychodramatische Arbeit und verrät den LeserInnen, welche Interventionen sie anwendet, um die Rollenflexibilität ihrer KlientInnen zu erhöhen und einen Prozess der Nachreifung zu ermöglichen.

In ihrem Beitrag beschreibt Kristina Scheuffgen ihre psychodramatherapeutische Arbeit mit Jugendlichen, die aufgrund von sexuellen Übergriffen in einer darauf spe- 
zialisierten Einrichtung untergebracht sind. Nachdem sie definiert, wann von sexuellen Übergriffen unter Jugendlichen gesprochen wird und welche familiäre Faktoren sexuelle Übergriffe begünstigen, lädt sie uns ein, sie bei einigen Sitzungen zu begleiten und gibt dabei Einblick in ihre an das Kinderpsychodrama nach Aichinger und Holl angelehnte, beachtenswerte Vorgehensweise. Die in diesem Setting von den Jugendlichen entwickelte, gespielte und aus der eigenen Perspektive nacherzählte Geschichte ermöglicht Schlussfolgerungen über die Störungsbilder der einzelnen Teilnehmer zu ziehen. Daraus werden Strategien für einzeltherapeutische und pädagogische Interventionen entwickelt, die häufig eine nachnährende, stabilisierende und selbstwertsteigernde Zielsetzung haben.

In der Rubrik „Der andere Artikel“ betont die Frauenärztin Mechthild Neises die Bedeutung einer genderspezifischen Herangehensweise bei der Behandlung von an psychosomatischen Erkrankungen leidenden Personen. In der Einleitung beschreibt sie, wie die Sichtweisen vergangener Tage vom Wesen von Frauen und Männern unsere inneren Bilder heute noch prägen und Auswirkungen auf die Stellung von Frauen und Männern in der Gesellschaft haben. Im spezifischen Teil zeigt sie - belegt durch zahlreiche Studien -, dass die psychosomatischen Problemfelder, die ihr in ihrer gynäkologischen Praxis begegnen, nur durch gendersensible Interventionen gelöst werden können. Auch in der Psychotherapie fordert sie, dass ein größerer Fokus auf geschlechtsspezifische Aspekte gelegt wird.

Die beiden Nachrufe auf Friedel Geisler und Heika Straub machen den Verlust zweier bedeutender deutscher Psychodramatikerinnen deutlich. Christiane Schlüters Bericht von der Psychodrama-Bühneneröffnung im Kommunikationszentrum in Goslar öffnet dagegen eher den Blick nach vorn. Ursula Hauser bedient mit ihrem Artikel Psychodrama in Costa Rica die Rubrik Vernetzung.

Wir möchten an dieser Stelle auch auf einige Neuerungen in der Redaktion hinweisen. Reinhard Krüger hat seine Aufgabe als Herausgeber der ZPS, die er dankenswerterweise kurzfristig übernommen hatte, an Sabine Spitzer übergeben. Damit ist die Herausgabe der ZPS binational geworden, was auch der Verbreitung des Psychodramas entspricht. Sabine Spitzer gehört der Redaktion bereits einige Jahre an, und ist den ZPS-LeserInnen auch als Autorin bekannt. Sie steht für einen Generationenwechsel in der Redaktion. Reinhard Krüger bleibt Redaktionsmitglied und wird weiterhin mit seinen Ideen zum Gelingen der Zeitschrift beitragen können. Verabschieden mussten wir jedoch Manfred Gellert, dem wir für sein langjähriges Engagement recht herzlich danken. Er ist als Heftherausgeber bereits 2005 positiv in Erscheinung getreten und hat mit seinem umfangreichen Wissen und Netzwerk der ZPS viele interessante Impulse gegeben. Wir haben aber auch einen Neuzugang zu verzeichnen: Michael Wieser ist seit Herbst 2011 ständiges Mitglied der Redaktion. Er ist vielen LeserInnen bekannt durch seine Aktivitäten für die Anerkennung des Psychodramas in Österreich im Rahmen des ÖAGG, aber auch als global keyplayer im internationalen Psychodrama. 